\title{
Correction to: Boundary Integral Formula for Harmonic Functions on Riemann Surfaces
}

\section{Peter L. Polyakov ${ }^{1}$}

Received: 22 August 2021 / Accepted: 31 August 2021 / Published online: 29 September 2021

(c) Springer-Verlag GmbH Germany, part of Springer Nature 2021

\section{Abstract}

We present a corrected proof of Proposition 2.3 in the article "Boundary integral formula for harmonic functions on Riemann surfaces" [2].

Keywords Riemann surfaces · Harmonic functions

Mathematics Subject Classification Primary 30F · 32A10 · 32A26

\section{Correction to: Comput. Methods Funct. Theory https://doi.org/10.1007/s40315-020-00308-x}

Let $\widetilde{V}$ be a compact Riemann surface and let

$$
V=\widetilde{V} \backslash\left(\bigcup_{r=1}^{m} V_{r}\right)
$$

be a subdomain in $\widetilde{V}$, where $\left\{V_{r}\right\}_{r=1}^{m}$ is a collection of disjoint neighborhoods with smooth boundaries. In [2, Prop. 2.3], the author claimed that for a specially embedded $V \subset \mathbb{C P}^{2}$ and an arbitrary harmonic function $u \in C^{1}(\bar{V})$ there exists a harmonic function $h \in C^{1}(\bar{V})$ such that

$$
\int_{b V_{r}} \partial(u-h)=0 \text { for } r=1, \ldots, m,
$$

Communicated by Dmitri Khavinson.

The original article can be found online at https://doi.org/10.1007/s40315-020-00308-x.

Peter L. Polyakov

polyakov@uwyo.edu

1 University of Wyoming, Laramie, USA 
where $\partial$ is the operator of holomorphic differentiation. As substantiating evidence the author suggested the existence of harmonic functions with log-type singularities at the points $z^{(r)} \in \widetilde{V} \backslash V, r=1, \ldots, m$. However, this argument on its own is not sufficient, and in the present note we provide additional arguments to complete the proof. In the proposition below, we prove the existence of a representation satisfying (2) for a general Riemann surface of type (1).

Proposition 1 Let $V$ be a smoothly bounded Riemann surface as in (1). Then there exists a set of real-valued harmonic functions $\left\{h_{j}\right\}_{j=1}^{m-1} \in C^{1}(\bar{V})$ such that for any harmonic function $u \in C^{1}(\bar{V})$ there exist coefficients $\left\{a_{j}\right\}_{j=1}^{m-1} \in \mathbb{C}$ satisfying

$$
\int_{b V_{r}} \partial\left(u-\sum_{j=1}^{m-1} a_{j} h_{j}\right)=0 \text { for } r=1, \ldots, m
$$

The formulas in the original article [2], which refer to Proposition 2.13 need also to be corrected. In particular, the final formula (1.12) and formula (3.6) should be replaced with the formula

$u\left(w^{(k)}\right)=\frac{1}{d+1} \cdot \operatorname{Re}\left\{\frac{w_{0}^{(k)} \cdot \operatorname{det} A_{k}[g]\left(w, w^{(1)}, \ldots, w^{(d-1)}\right)}{\operatorname{det} A(w)}\right\}+\sum_{j=1}^{m-1} a_{j} h_{j}\left(w^{(k)}\right)$

where $\left\{h_{j}\right\}_{1}^{m-1}$ are fixed harmonic functions with log-type singularities at selected fixed points in $\widetilde{V} \backslash V$, and the coefficients $\left\{a_{j}\right\}_{1}^{m-1}$ depending on the form $\left.\partial u\right|_{b V}$ are constructed in the proof of Proposition 1.

The proof of Proposition 1 is based on the application of three lemmas below. In those lemmas we use O. Forster's idea from his book [1] to consider two special cases of construction of harmonic functions on open Riemann surfaces.

Lemma 1 Let $z^{(1)}, z^{(2)} \in U \Subset \widetilde{V}$, where $U$ is a coordinate neighborhood in $\widetilde{V}$ with coordinate function $\zeta$ such that $\zeta\left(z^{(1)}\right)=0, \zeta\left(z^{(2)}\right)=1 / 2$. Then there exist neighborhoods $U_{j} \ni z^{(j)}$ and a holomorphic function $f$ on $\widetilde{V} \backslash\left(U_{2} \cup z^{(1)}\right)$ such that

$$
\left.f\right|_{U_{1}}=\zeta^{-1} \cdot \phi
$$

where $\phi$ is a holomorphic function in $U_{1}$ with $\phi\left(z^{(1)}\right) \neq 0$, and

$$
\left\{\begin{array}{l}
\frac{1}{2 \pi i} \int_{b U_{1}} \partial \log |f(\zeta)|^{2}=-1 \\
\frac{1}{2 \pi i} \int_{b U_{2}} \partial \log |f(\zeta)|^{2}=1 .
\end{array}\right.
$$


Proof We consider a smooth function $\psi$ such that

$$
\psi(\zeta)= \begin{cases}1 & \text { if }|\zeta|<r_{1} \text { for some } r_{1} \in\left(\frac{3}{4}, 1\right) \\ 0 & \text { if }|\zeta|>r_{2} \text { for some } r_{2} \in\left(r_{1}, 1\right)\end{cases}
$$

and define the function $f_{0}$ on $U \backslash z^{(1)}$ by the formulas

$$
f_{0}(\zeta)= \begin{cases}\exp \left(\psi(\zeta) \cdot \log \left(\frac{\zeta-1 / 2}{\zeta}\right)\right) & \text { if } r_{1}<|\zeta|<1 \\ \frac{\zeta-1 / 2}{\zeta} & \text { if }|\zeta|<r_{1}\end{cases}
$$

where $\log ((\zeta-1 / 2) / \zeta)$ is a univalent branch of the $\log$, which is well defined for $\zeta$ satisfying $|\zeta| \in\left(r_{1}, 1\right)$. From the definition of the function $f_{0}$ it follows that it can be extended to $\widetilde{V} \backslash U$ as $f_{0}(\zeta)=1$.

We consider the neighborhoods

$$
\begin{aligned}
U_{1} & =\left\{\zeta \in U:|\zeta|<\frac{1}{4}\right\}, \\
U_{2} & =\left\{\zeta \in U:\left|\zeta-\frac{1}{2}\right|<\frac{1}{8}\right\}, \\
U_{2}(\epsilon) & =\left\{\zeta \in U:\left|\zeta-\frac{1}{2}\right|<\frac{1}{8}-\epsilon\right\} \Subset U_{2}, \\
W & =\widetilde{V} \backslash\left(\bar{U}_{2}(\epsilon) \cup\left\{|\zeta| \leq \frac{1}{8}\right\}\right),
\end{aligned}
$$

satisfying the following equalities

$$
\begin{aligned}
& U_{1} \cup W=\widetilde{V} \backslash \bar{U}_{2}(\epsilon), \\
& U_{1} \cap W=\left\{\zeta \in U: \frac{1}{8}<|\zeta|<\frac{1}{4}\right\} .
\end{aligned}
$$

To construct a meromorphic function on $\widetilde{V} \backslash \bar{U}_{2}$ satisfying (5) we consider the smooth differential form $\alpha^{(0,1)}$

$$
\begin{array}{ll}
\alpha^{(0,1)}=\frac{\bar{\partial} f_{0}}{f_{0}} & \text { on } W, \\
\alpha^{(0,1)}=0 & \text { on } U_{1} .
\end{array}
$$

Using the solvability of the $\bar{\partial}$-equation on the open Riemann surface $\widetilde{V} \backslash \bar{U}_{2}(\epsilon)$ (see [1]) we obtain a smooth function $g$ such that $\bar{\partial} g=\alpha^{(0,1)}$ and the meromorphic function $f(z)=e^{-g} \cdot f_{0}$ on $\widetilde{V} \backslash \bar{U}_{2}(\epsilon)$ having the only pole at $z^{(1)}$ and no zeros, and satisfying on $\widetilde{V} \backslash\left(\bar{U}_{2}(\epsilon) \cup z^{(1)}\right)$ the condition 


$$
\bar{\partial} f=-e^{-g} f_{0} \bar{\partial} g+e^{-g} \bar{\partial} f_{0}=e^{-g} \cdot f_{0}\left(-\bar{\partial} g+\frac{\bar{\partial} f_{0}}{f_{0}}\right)=0 .
$$

From (8) we obtain that for $\zeta \in U,|\zeta|<r_{1}$ we have

$$
\log |f(\zeta)|^{2}=\log \left|e^{-g(\zeta)}\right|^{2}+\log \left|\zeta-\frac{1}{2}\right|^{2}-\log |\zeta|^{2}
$$

and therefore

$$
\begin{aligned}
& \frac{1}{2 \pi i} \int_{b U_{1}} \partial \log |f(\zeta)|^{2} \\
& \quad=\frac{1}{2 \pi i}\left(\int_{b U_{1}} \partial \log \left|e^{-g(\zeta)}\right|^{2}+\int_{b U_{1}} \partial \log \left|\zeta-\frac{1}{2}\right|^{2}-\int_{b U_{1}} \partial \log |\zeta|^{2}\right) \\
& \quad=\frac{1}{2 \pi i}\left(\int_{b U_{1}} \frac{-e^{-\bar{g}(\zeta)} e^{-g(\zeta)}}{\left|e^{-g(\zeta)}\right|^{2}} \partial g(\zeta)+\int_{b U_{1}} \frac{\bar{\zeta}-\frac{1}{2}}{\left|\zeta-\frac{1}{2}\right|^{2}} d \zeta-\int_{b U_{1}} \frac{\bar{\zeta}}{|\zeta|^{2}} d \zeta\right) \\
& \quad=\frac{1}{2 \pi i}\left(-\int_{b U_{1}} \partial g(\zeta)+\int_{b U_{1}} \frac{d \zeta}{\zeta-\frac{1}{2}}-\int_{b U_{1}} \frac{d \zeta}{\zeta}\right) .
\end{aligned}
$$

Since $g$ is holomorphic in $\left\{\zeta \in U:|\zeta|<r_{1}\right\} \backslash \bar{U}_{2}(\epsilon)$, we have $\partial g=d g$, and therefore

$$
\int_{b U_{1}} \partial g(\zeta)=\int_{b U_{1}} d g(\zeta)=0
$$

Similarly, we have

$$
\int_{b U_{1}} \frac{d \zeta}{(\zeta-1 / 2)}=0 \text { and } \int_{b U_{1}} \frac{d \zeta}{\zeta}=2 \pi i
$$

Then using the equalities above and similar equalities for $\int_{b U_{2}}$ we obtain equalities (6).

Lemma 2 Let $U_{0} \Subset \widetilde{V}$ be an open neighborhood in $\widetilde{V}$, and let $z^{(1)}, z^{(2)} \in U \Subset \widetilde{V} \backslash \bar{U}_{0}$, where $U$ is a coordinate neighborhood in $\widetilde{V}$ with coordinate function $\zeta$ such that $\zeta\left(z^{(1)}\right)=0, \zeta\left(z^{(2)}\right)=1 / 2$. Then there exist neighborhoods $U_{j} \ni z^{(j)}, j=1,2$, and a meromorhic function $f$ on $\widetilde{V} \backslash\left(\bar{U}_{0} \cup z^{(1)} \cup z^{(2)}\right)$ such that

$$
\left\{\begin{array}{l}
\frac{1}{2 \pi i} \int_{b U_{1}} \partial \log |f(\zeta)|^{2}=-1, \\
\frac{1}{2 \pi i} \int_{b U_{2}} \partial \log |f(\zeta)|^{2}=1 .
\end{array}\right.
$$


Proof As in the proof of Lemma 1 we consider a smooth function $\psi$ satisfying conditions (7) and define the function $f_{0}$ on $U \backslash\left(z^{(1)} \cup z^{(2)}\right)$ by (8). Again from the definition of the function $f_{0}$ it follows that it can be extended to $\widetilde{V} \backslash U$ as $f_{0}(\zeta)=1$.

We consider the neighborhoods

$$
\begin{aligned}
U_{1} & =\left\{\zeta \in U:|\zeta|<\frac{1}{4}\right\}, \\
U_{2} & =\left\{\zeta \in U:\left|\zeta-\frac{1}{2}\right|<\frac{1}{4}\right\}, \\
W & =\widetilde{V} \backslash\left(\bar{U}_{0} \cup\left\{|\zeta| \leq \frac{1}{8}\right\} \cup\left\{\left|\zeta-\frac{1}{2}\right| \leq \frac{1}{8}\right\}\right),
\end{aligned}
$$

satisfying the following equalities

$$
\begin{aligned}
& \left(U_{1} \cup U_{2}\right) \cup W=\widetilde{V} \backslash \bar{U}_{0}, \\
& \left(U_{1} \cup U_{2}\right) \cap W=\left\{\zeta \in U: \frac{1}{8}<|\zeta|<\frac{1}{4}, \frac{1}{8}<\left|\zeta-\frac{1}{2}\right|<\frac{1}{4}\right\} .
\end{aligned}
$$

To construct a meromorphic function on $\widetilde{V} \backslash \bar{U}_{0}$ satisfying (12) we consider the smooth differential form $\alpha^{(0,1)}$

$$
\begin{cases}\alpha^{(0,1)}=\frac{\bar{\partial} f_{0}}{f_{0}} & \text { on } W \\ \alpha^{(0,1)}=0 & \text { on }\left(U_{1} \cup U_{2}\right)\end{cases}
$$

Then on the open Riemann surface $\widetilde{V} \backslash \bar{U}_{0}$ we consider a smooth function $g$ such that $\bar{\partial} g=\alpha^{(0,1)}$ and the meromorphic function $f(z)=e^{-g} \cdot f_{0}$ on $\widetilde{V} \backslash\left(\bar{U}_{0} \cup z^{(1)} \cup z^{(2)}\right)$ satisfying

$$
\bar{\partial} f=-e^{-g} f_{0} \bar{\partial} g+e^{-g} \bar{\partial} f_{0}=e^{-g} \cdot f_{0}\left(-\bar{\partial} g+\frac{\bar{\partial} f_{0}}{f_{0}}\right)=0 .
$$

From (8) we obtain that for $\zeta \in U,|\zeta|<r_{1}$ we have

$$
\log |f(\zeta)|^{2}=\log \left|e^{-g(\zeta)}\right|^{2}+\log \left|\zeta-\frac{1}{2}\right|^{2}-\log |\zeta|^{2}
$$

and therefore, as in (11)

$$
\frac{1}{2 \pi i} \int_{b U_{1}} \partial \log |f(\zeta)|^{2}=\frac{1}{2 \pi i}\left(-\int_{b U_{1}} \partial g(\zeta)+\int_{b U_{1}} \frac{d \zeta}{\zeta-\frac{1}{2}}-\int_{b U_{1}} \frac{d \zeta}{\zeta}\right)
$$


Since $g$ is holomorphic in $\left\{\zeta \in U:|\zeta|<r_{1}\right\} \backslash\left(U_{1} \cup U_{2}\right)$, we have $\partial g=d g$, and therefore

$$
\left\{\begin{array}{l}
\int_{b U_{1}} \partial g(\zeta)=\int_{b U_{1}} d g(\zeta)=0 \\
\int_{b U_{2}} \partial g(\zeta)=\int_{b U_{2}} d g(\zeta)=0 .
\end{array}\right.
$$

Using the equalities above together with equalities

$$
\int_{b U_{1}} \frac{d \zeta}{\zeta-\frac{1}{2}}=\int_{b U_{2}} \frac{d \zeta}{\zeta}=0
$$

we obtain equalities (12).

The following statement formulated in the terminology of the book [1] is a corollary of Lemma 2:

Corollary 1 Any divisor $D$ with $\operatorname{deg} D=0$ on an open Riemann surface is solvable.

Lemma 3 Let $z_{\widetilde{\sim}}^{(1)}, z^{(2)}$ be two points in $\widetilde{V}$, let $U_{j} \ni z^{(j)}$ be two neighborhoods of those points in $\widetilde{V}$, and let $U_{j}(\epsilon) \subset U_{j}$ be slightly smaller neighborhoods. Then there exists a holomorphic function $f$ on $\widetilde{V} \backslash\left(\bar{U}_{1}(\epsilon) \cup \bar{U}_{2}(\epsilon)\right)$ such that

$$
\left\{\begin{array}{l}
\frac{1}{2 \pi i} \int_{b U_{1}} \partial \log |f(\zeta)|^{2}=-1 \\
\frac{1}{2 \pi i} \int_{b U_{2}} \partial \log |f(\zeta)|^{2}=1
\end{array}\right.
$$

Proof For the points $z^{(1)}, z^{(2)}$ we consider a sequence of points $z^{(1)}=w^{(1)}, \ldots, w^{(r)}=$ $z^{(2)}$ such that every two consecutive points in the sequence belong to the same coordinate neighborhood. Applying Lemma 1 we construct two meromorphic functions: $g_{1}$ on $\widetilde{V} \backslash \bar{U}_{1}(\epsilon)$ with zero at $w^{(2)}$ and $g_{r-1}$ on $\widetilde{V} \backslash \bar{U}_{2}(\epsilon)$ with pole at $w^{(r-1)}$. Then using Lemma 2 we construct a sequence of meromorphic functions $g_{j}, j=2, \ldots, r-2$, on $\widetilde{V} \backslash\left(\bar{U}_{1}(\epsilon) \cup \bar{U}_{2}(\epsilon)\right)$ such that $g_{j}$ has a pole at $w^{(j)}$ and a zero at $w^{(j+1)}$. Defining then

$$
f=\prod_{j=1}^{r-1} g_{j}
$$

we obtain the desired function.

Proof of Proposition 1

Proof Using Lemma 3 we construct a set of holomorphic functions $\left\{f_{1}, \ldots, f_{m-1}\right\}$ satisfying (15) on $\widetilde{V} \backslash\left(V_{r} \cup V_{r+1}\right)$, and define functions $h_{r}=\log \left|f_{r}\right|^{2}$. Then we prove 
the existence for an arbitrary harmonic $u \in C^{1}(\bar{V})$ of the coefficients satisfying (3). Let $l \in \mathbb{Z}$ be the number such that there exist coefficients $a_{j}$ for $j>l$ satisfying

$$
\int_{b V_{j}} \partial\left(u-\sum_{l+1}^{m-1} a_{j} h_{j}\right)=0 \text { for } j=l+2, \ldots, m \text {. }
$$

We prove the proposition by induction with respect to $l$. Namely, assuming that for some $l$ equality (16) is satisfied for $j>l+1$, we define

$$
a_{l}=\int_{b V_{l+1}} \partial\left(u-\sum_{l+1}^{m-1} a_{j} h_{j}\right)
$$

and obtain that equality

$$
\int_{b V_{j}} \partial\left(u-\sum_{l}^{m-1} a_{j} h_{j}\right)=0
$$

is satisfied for $j \geq l+1$.

\section{References}

1. Forster, O.: Lectures on Riemann Surfaces. Springer, New York (1981)

2. Polyakov, P.: Boundary integral formula for harmonic functions on Riemann surfaces. Comput. Methods Funct. Theory 20(2), 235-253 (2020)

Publisher's Note Springer Nature remains neutral with regard to jurisdictional claims in published maps and institutional affiliations. 